\title{
DISTANCE EDUCATION IN MEDICAL SCHOOLS: THE EXPERIENCE AND OPINIONS OF ACADEMICIANS AND STUDENTS
}

\author{
Hilal Sena Çifcibaşı ${ }^{1}$ Berkay $\operatorname{Kef}^{2}$, Irmak İrem Özyiğit ${ }^{1}$, Sezin Sayın ${ }^{1}$, Aslı Göztepe ${ }^{1}$, Selis Gülseven Güven ${ }^{3}$, \\ Serkan $\mathrm{Atic1}^{4}$ \\ ${ }^{1}$ Trakya University School of Medicine, Edirne, TURKEY \\ ${ }^{2}$ Okan University School of Medicine, Istanbul, TURKEY \\ ${ }^{3}$ Department of Ear-Nose and Throat Surgery, Trakya University School of Medicine, Edirne, TURKEY \\ ${ }^{4}$ Division of Pediatric Infectious Diseases, Department of Pediatrics, Okan University School of Medicine, Istanbul, \\ TURKEY
}

\begin{abstract}
Aims: This study aims to identify the thoughts and opinions of medical schools students and academicians about the distance medical education and examination system that has been rapidly applied during the COVID-19 pandemic Methods: A questionnaire was prepared via Google Forms which consisted of a total of 3 sections. Informed consent was obtained in the first section and participants were directed to "student" or "academician" section. There were 28 questions for the students and 24 questions for the academicians. Categorical variables were demonstrated as numbers and percentages, whereas continuous variables were presented as minimum, maximum, and mean values. Chi-squared test was used to compare preclinic and clinic year students, and the academicians in preclinic, medical, and surgical fields. Results: A total of 321 participants completed the questionnaire. The mean participant ages were 21.4 years and 41.68 years for the students and the academicians, respectively. Only $30 \%$ of the students thought the distance education lessons were beneficial while it was $35.5 \%$ for the academicians. $25.8 \%$ of the academicians and $29.6 \%$ of the students were indecisive on the matter. When the examination process was taken into account $67.7 \%$ of the academicians and $56.9 \%$ of the students thought the online examinations were not reliable. Conclusion: It is predicted that the pandemic process will continue in the next academic years. Considering the current situation, distance education seems to be the best option to ensure that the learning process can continue while protecting the health of students and academicians. Although distance education is not sufficient by itself in medical education during and after the COVID-19 pandemic, it is a method that should be used in almost every field of medical education, especially in the preclinical phase. Both the distance education, and online examination process require improvements and they are needed to be supported with face to face lectures and practices.Keywords: COVID-19, academician, medical student, distance learning, pandemic
\end{abstract}

\section{INTRODUCTION}

Coronavirus disease-19 (COVID-19) has made a definite entry into our lives since December 31, 2019 and continues to show its effects. With this entry viral diseases have been on the agenda of governments all across the world as well as the medical world (1).

Viruses, especially SARS-CoV-2, which increase and show up and affect the whole world, will obviously be in our lives for a while and we have to adapt our daily lives and most importantly education systems into the new normal (2).
Coronavirus disease-19 outbreak caused serious changes in the education field in Turkey, as well as all over the world. Upon the announcement of the first case in Turkey on March 10, 2020, all face-to-face education activities across the country were stopped as of March 16, and subsequently, online/distance education processes were initiated (3).

The primary purpose of this was to ensure the isolation of students and education staff, such as a very important part of the population, and to control the pandemic as much as possible (2). 
The necessity of making all these changes during the school period and without interrupting the education created difficulties in terms of both time constraints and feasibility. Online education is challenging, especially in education programs where practical applications are as important as theoretical applications such as medical faculties (4).

Considering that online education depends on the center, the instructor and of course the participation of the students, it is not possible to talk about a standard for now. Access to technological devices and internet, financial problems are among the issues that challenge students (5).

In addition, the fact that the practical training that medical students will receive takes place in the hospital makes the situation even more difficult as it requires the educators to pay attention to the safety of the students while providing care to the patients (5).

It is predicted that the pandemic process will continue in the next academic years (1). For this reason, determining the thoughts and opinions of medical faculty students and academicians about online education and the examination process is of great importance to increase the quality of education $(5,6)$. In this cross-sectional study, we aimed to identify the thoughts and opinions of medical students and academicians about the online education and examination system that has been rapidly passed through the COVID-19 pandemic process.

\section{MATERIAL AND METHODS}

This study was approved by the Scientific Research Ethics Committee of Trakya University School of Medicine (Protocol Code: TÜTF-BAEK 2020/343). This descriptive study was carried out between September and October 2020. Individuals other than medical students and medical school academicians were not included in the study. The study was conducted via a self-administrative online questionnaire in the Turkish language and delivered through scientific research communities of the medical schools. Information about the study was provided at the beginning of the questionnaire and the participants' consent was required to continue forward.

The questionnaire was prepared via Google Forms and consisted of a total of 3 sections. Informed consent was obtained in the first section and participants were directed to "student" or "academician" section. There were 28 questions for the students and 24 questions for the academicians (Table 1).
The age, gender, years in university, the name of the university, and chronic diseases of the participants were questioned in both groups for demographics. Categorical variables are demonstrated as numbers and percentages, whereas continuous variables are presented as the minimum, maximum, and mean values. The IBM SPSS version 23 was used for the statistical analysis of the data. Chi-squared test was used to compare preclinic and clinic year students, and the academicians in preclinic, medical, and surgical fields. A p-value $<0.05$ was set for statistical significance.

\section{RESULTS}

This questionnaire-based study was conducted among 290 medical students, and 31 academicians. Medical students are composed of 165 (56.9\%) preclinical, and 125 (43.1\%) clinical students from 15 different universities. The highest participation was from Trakya University with a rate of $69.1 \%$. The mean of the students' age was 21.4 years (standard deviation [SD]: 2.267 years, range: 17-38 years). One hundred and eighty-two (62.8\%) were female, and 108 (37.2\%) were male. The dispersion of the students' grades is shown in Table 2. Thirty-one medical school academicians from ten different universities in Turkey participated in the online survey. The mean of the academicians' age was 41.68 years (SD: 7.512 years, range: $28-56$ years). Eighteen (58.1\%) were female, and 13 (41.9\%) were male. The specialties, academic titles, and academic experiences (in years) of the academicians are shown in Table 3.

The majority of the students had no chronic disease (89.7\%), however, 14 (4.8\%) had asthma, 2 (0.7\%) had diabetes, and 14 (4.8\%) of them had some other chronic diseases. The number of academicians with no chronic disease was 23 (74.2\%), and 1 (3.2\%) of them had asthma, 1 (3.2\%) of them had diabetes, and 6 (19.4\%) of them had some other chronic diseases.

Of the students, two hundred and fifty-five (87.6\%) used computers, 96 (33\%) used smartphones, and 34 (11.7\%) used tablets to follow their distance education lessons.

The leading problem students encountered was not having the proper study environment (46.7\%) while this was not the leading problem for the academicians $(12.9 \%)$. About $35.5 \%$ of the academicians had internet access problems, technological inefficacy, and systemic problems. One hundred and twenty (41.2\%) students experienced internet access problems, 112 (38.5\%) stu- 
dents had systemic issues, and 95 (32.6\%) had technological inefficacy. Eighty-nine (30.6\%) students did not have proper time while only $4(12.9 \%)$ academicians had no proper time during the distance education process.

The thoughts of medicals students' and academicians' on distance learning are shown in Tables 4 and 5, respectively. According to $40.3 \%$ of the students, distance education lessons are not beneficial and academicians are in co-decision with this answer. Also, $66.6 \%$ (40.7\% do not agree at all, $25.9 \%$ do not agree) of the students think that distance education lessons are not useful as face-to-face lessons. Of the academicians, $45.2 \%$ who are in medical fields think that they are not as useful as face-to-face lessons. About $87.1 \%$ of the academicians think that distance education alone is insufficient in the field of medicine. By $43.1 \%$ do not agree at all, and $21.0 \%$ do not agree answers, medical students think that it is inconvenient to include interns in distance learning. Twenty-five (80.6\%) academicians also think that interns should not be included in distance education. With the majority of 26 academicians, "practical lessons are not suitable" was the most common answer.

According to Table 6, 63 (38.2\%) preclinical and $71(56.8 \%)$ clinical students think that distance education was inadequate alone for the basic sciences, but most of them should be given as distance education, and this was statistically significant $(\mathrm{p}<0.001)$. Eightyfour $(50.9 \%)$ preclinical students think that practical lessons in distance education were completely insufficient alone, and all of them should be in-class lessons, while $55(44.0 \%)$ clinical students think that they are inadequate alone, and most of them should be faceto-face, and these results were statistically significant $(\mathrm{p}=0.011)$.

The overall responses for the user experience of distance learning applications are presented in Tables 7 and 8. Students predominately think that accessing the course was easy $(n=182,62.7 \%)$. One hundred and seventy-five (60.3\%) students said that if they did not attend a live lecture, they can easily access and watch the recordings, and accessing the course contents is quick for the 196 (67.6\%) students. Of the students, 125 (43.1\%) occasionally encounter technical problems in virtual classroom applications, however, 182 (62.8\%) of them find the distance education system not complicated. Twelve (38.7\%) academicians know how to solve the problems they encountered and
$12(38.7 \%)$ were indecisive. For the question of whether the distance education lessons are interactive enough, 21 (49.8\%) academicians think that they were not.

The medical students' and academicians' thoughts on online examinations are shown in Table 9. There was a statistically significant association between the academicians' answers on whether the online exams measure learning or not $(\mathrm{p}=0.000239)$. On the question of online exams' reliability, a statistically significant difference was found $(\mathrm{p}=0.003)$. None of the academicians chose the "do not agree at all" option for the question on whether they think that the students cheat in the online exam. Also, the answers to this question were statistically significant $(\mathrm{p}=0.027)$. 
Table 1: The questionnaire used in the study.

\begin{tabular}{l} 
Questions \\
\hline For Medical Students and Academicians \\
\hline 1) Do you have any chronic illnesses? \\
2) I think distance education lessons are beneficial \\
3) I find distance education alone sufficient in the \\
field of medicine \\
4) I think distance education lessons are as useful \\
as face-to-face lessons \\
5) I find it convenient to include interns in \\
distance education \\
6) What do you think about the competence of \\
distance education for basic sciences?
\end{tabular}

7) What do you think about the competence of distance education for clinical sciences?

8) Apart from the pandemic, I think theoretical lessons can be given in the form of distance education

9) I would like distance education to be available also outside of working hours

10) Theoretical courses are suitable for distance education

11) Practical lessons are suitable for distance education

12) What kind of problems did you encounter during the distance education process?

13) I know how to solve the problems I encounter in the distance education system

14) I occasionally encounter technical problems in virtual classroom application

15) The distance education system seems very complicated to me

16) I think online exams measure learning

17) I think online exams are reliable
Answers

Asthma / COPD / Diabetes / Chronic renal failure / Other / None

Do not agree at all / Do not agree / Indecisive / Agree / Completely agree

Do not agree at all / Do not agree / Indecisive / Agree / Completely agree

Do not agree at all / Do not agree / Indecisive / Agree / Completely agree

Do not agree at all / Do not agree / Indecisive / Agree / Completely agree

Alone enough / Inadequate alone, but most of them should be distance education / Inadequate alone, and most of them should be in-class education / Completely insufficient alone, all of them should be in-class education

Alone enough / Inadequate alone, but most of them should be distance education / Inadequate alone, and most of them should be in-class education / Completely insufficient alone, all of them should be in-class education

Do not agree at all / Do not agree / Indecisive / Agree / Completely agree

Do not agree at all / Do not agree / Indecisive / Agree / Completely agree

Do not agree at all / Do not agree / Indecisive / Agree / Completely agree

Do not agree at all / Do not agree / Indecisive / Agree / Completely agree

Internet access / Technological inefficacy / Not having the proper study environment / Not having the proper time / Systemic issues / Other

Do not agree at all / Do not agree / Indecisive / Agree / Completely agree

Do not agree at all / Do not agree / Indecisive / Agree / Completely agree

Do not agree at all / Do not agree / Indecisive / Agree / Completely agree

Do not agree at all / Do not agree / Indecisive / Agree / Completely agree

Do not agree at all / Do not agree / Indecisive / Agree /

For Medical Students Only

18) Which university do you study at?

19) Which class are you currently enrolled?

20) On which devices do you follow distance education lessons?

21) I regularly attend distance education lessons

22) I can easily access the course I want in the distance education system

23) I can easily access and watch the recordings of the live lectures I did not attend

24) The educational materials used in the lessons meet my needs

25) I can quickly access the course contents

26) I know how to use virtual classroom application

27) I can easily find the information I want in the distance education system

28) I think the information (announcement etc.) is sufficient

For Academicians Only

29) What is your specialty?

30) Which university do you teach at?

31) Academic title

32) Academic experience (in years)

33) I can use the virtual classroom application

without support

34) I think the distance education lessons are

interactive enough

35) I think the students cheat in the online exams
Completely agree

\section{Prep / $1 / 2 / 3 / 4 / 5 / 6$ \\ Computer / Smartphone / Tablet / Other}

Do not agree at all / Do not agree / Indecisive / Agree / Completely agree

Do not agree at all / Do not agree / Indecisive / Agree / Completely agree

Do not agree at all / Do not agree / Indecisive / Agree / Completely agree

Do not agree at all / Do not agree / Indecisive / Agree / Completely agree

Do not agree at all / Do not agree / Indecisive / Agree / Completely agree

Do not agree at all / Do not agree / Indecisive / Agree / Completely agree

Do not agree at all / Do not agree / Indecisive / Agree / Completely agree

Do not agree at all / Do not agree / Indecisive / Agree / Completely agree

\section{Preclinical / Medical / Surgical}

Guest teaching assistant / Teaching assistant / Associate professor / Professor $0-5 / 6-10 / 11-15 / 16-20 / 20+$

Do not agree at all / Do not agree / Indecisive / Agree / Completely agree

Do not agree at all / Do not agree / Indecisive / Agree / Completely agree

Do not agree at all / Do not agree / Indecisive / Agree / 
Table 2: Medical students' demographics.

\begin{tabular}{cc} 
Grades & Number of Students [n (\%)] \\
\hline $\mathbf{1}$ & $15(5.2)$ \\
$\mathbf{2}$ & $88(30.3)$ \\
$\mathbf{3}$ & $62(21.4)$ \\
$\mathbf{4}$ & $48(16.6)$ \\
$\mathbf{5}$ & $42(14.5)$ \\
$\mathbf{6}$ & $35(12.1)$ \\
Total & $290(100.0)$ \\
\hline
\end{tabular}

Table 3: Demographics of academicians.

Number of Academicians [n (\%)]

\begin{tabular}{lc}
\hline Specialty & $2(6.5)$ \\
Preclinical & $24(77.4)$ \\
Medical & $5(16.1)$ \\
Surgical & \\
Academic title & $4(12.9)$ \\
Guest teaching assistant & $19(61.3)$ \\
Teaching assistant & $4(12.9)$ \\
Associate professor & $4(12.9)$ \\
Professor & \\
Academic experience & $18(58.1)$ \\
0-5 years & $3(9.7)$ \\
6-10 years & $4(12.9)$ \\
11-15 years & $3(9.7)$ \\
16-20 years & $3(9.7)$ \\
20+ years & $31(100.0)$ \\
Total & \\
\hline
\end{tabular}


Table 4: Medical Students' thoughts on distance learning.

\begin{tabular}{|c|c|c|c|c|c|c|}
\hline & $\begin{array}{c}\text { Do not } \\
\text { agree at all } \\
\text { n (\%) }\end{array}$ & $\begin{array}{c}\text { Do not } \\
\text { agree } \\
\text { n (\%) }\end{array}$ & $\begin{array}{l}\text { Indecisive } \\
\text { n (\%) }\end{array}$ & $\begin{array}{l}\text { Agree } \\
\text { n (\%) }\end{array}$ & $\begin{array}{l}\text { Completely } \\
\text { agree } \\
\text { n (\%) }\end{array}$ & $\begin{array}{c}\text { P- } \\
\text { value } \\
*\end{array}$ \\
\hline I regularly attend distance education & & & & & & 0.354 \\
\hline lessons & & & & & & \\
\hline Preclinical Students & $4(1.4)$ & $10(3.4)$ & $26(9.0)$ & $85(29.3)$ & $40(13.8)$ & \\
\hline Clinical Students & $2(0.7)$ & $10(3.4)$ & $14(4.8)$ & $57(19.7)$ & $42(14.5)$ & \\
\hline Distance education lessons are beneficial & & & & & & 0.041 \\
\hline Preclinical Students & $26(9.0)$ & $40(13.8)$ & $54(18.6)$ & $29(10.0)$ & $16(5.5)$ & \\
\hline Clinical Students & $13(4.5)$ & $38(13.1)$ & $32(11.0)$ & $36(12.4)$ & $6(2.1)$ & \\
\hline $\begin{array}{l}\text { Distance education alone is sufficient in } \\
\text { the field of medicine }\end{array}$ & & & & & & 0.905 \\
\hline Preclinical Students & $107(36.9)$ & $30(10.3)$ & $13(4.5)$ & $6(2.1)$ & $9(3.1)$ & \\
\hline Clinical Students & $79(27.2)$ & $23(7.9)$ & $11(3.8)$ & $7(2.4)$ & $5(1.7)$ & \\
\hline $\begin{array}{l}\text { Distance education lessons are useful as } \\
\text { face-to-face lessons }\end{array}$ & & & & & & 0.023 \\
\hline Preclinical Students & $62(21.4)$ & $54(18.6)$ & $19(6.6)$ & $19(6.6)$ & $11(3.8)$ & \\
\hline Clinical Students & $56(19.3)$ & $21(7.2)$ & $19(6.6)$ & $23(7.9)$ & $6(2.1)$ & \\
\hline $\begin{array}{l}\text { It is convenient to include interns in } \\
\text { distance education }\end{array}$ & & & & & & 0.017 \\
\hline Preclinical Students & $74(25.5)$ & $36(12.4)$ & $36(12.4)$ & $17(5.9)$ & $2(0.7)$ & \\
\hline Clinical Students & $51(17.6)$ & $25(8.6)$ & $21(7.2)$ & $16(5.5)$ & $12(4.1)$ & \\
\hline $\begin{array}{l}\text { The theoretical courses are suitable for } \\
\text { distance education }\end{array}$ & & & & & & 0.133 \\
\hline Preclinical Students & $18(6.2)$ & $31(10.7)$ & $39(13.4)$ & $48(16.6)$ & $29(10.0)$ & \\
\hline Clinical Students & $6(2.1)$ & $17(5.9)$ & $27(9.3)$ & $49(16.9)$ & $26(9.0)$ & \\
\hline $\begin{array}{l}\text { Practical lessons are suitable for distance } \\
\text { education }\end{array}$ & & & & & & 0.362 \\
\hline Preclinical Students & $111(38.3)$ & $33(11.4)$ & $13(4.5)$ & $4(1.4)$ & $4(1.4)$ & \\
\hline Clinical Students & $73(25.2)$ & $31(10.7)$ & $17(5.9)$ & $2(0.7)$ & $2(0.7)$ & \\
\hline $\begin{array}{l}\text { Apart from the pandemic, theoretical } \\
\text { lessons can be given in the form of } \\
\text { distance education }\end{array}$ & & & & & & 0.108 \\
\hline Preclinical Students & $65(22.4)$ & $32(11.0)$ & $16(5.5)$ & $31(10.7)$ & $21(7.2)$ & \\
\hline Clinical Students & $31(10.7)$ & $26(9.0)$ & $18(6.2)$ & $28(9.7)$ & $22(7.6)$ & \\
\hline
\end{tabular}

${ }^{\star}$ Statistically significant values are marked as bold. 
Table 5: The academicians' thoughts on distance learning.

\begin{tabular}{|c|c|c|c|c|c|c|}
\hline & $\begin{array}{l}\text { Do not } \\
\text { agree at all } \\
\text { n (\%) }\end{array}$ & $\begin{array}{l}\text { Do not } \\
\text { agree } \\
\text { n (\%) }\end{array}$ & $\begin{array}{l}\text { Indecisive } \\
\text { n (\%) }\end{array}$ & $\begin{array}{l}\text { Agree } \\
\text { n (\%) }\end{array}$ & $\begin{array}{l}\text { Completely } \\
\text { agree } \\
\text { n (\%) }\end{array}$ & $\begin{array}{c}\text { P- } \\
\text { value } \\
*\end{array}$ \\
\hline Distance education lessons are beneficial & & & & & & 0.005 \\
\hline Preclinical academicians & $0(0.0)$ & $1(3.2)$ & $0(0.0)$ & $0(0.0)$ & $1(3.2)$ & \\
\hline Medical academicians & $1(3.2)$ & $9(29.0)$ & $8(25.8)$ & $6(19.4)$ & $0(0.0)$ & \\
\hline Surgical academicians & $0(0.0)$ & $1(3.2)$ & $0(0.0)$ & $4(12.9)$ & $0(0.0)$ & \\
\hline $\begin{array}{l}\text { Distance education alone is sufficient in } \\
\text { the field of medicine }\end{array}$ & & & & & & 0.007 \\
\hline Preclinical academicians & $1(3.2)$ & $0(0.0)$ & $0(0.0)$ & $0(0.0)$ & $1(3.2)$ & \\
\hline Medical academicians & $14(45.2)$ & $8(25.8)$ & $2(6.5)$ & $0(0.0)$ & $0(0.0)$ & \\
\hline Surgical academicians & $3(9.7)$ & $1(3.2)$ & $0(0.0)$ & $1(3.2)$ & $0(0.0)$ & \\
\hline $\begin{array}{l}\text { It is convenient to include interns in } \\
\text { distance education }\end{array}$ & & & & & & 0.002 \\
\hline Preclinical academicians & $1(3.2)$ & $0(0.0)$ & $0(0.0)$ & $0(0.0)$ & $1(3.2)$ & \\
\hline Medical academicians & $15(48.4)$ & $5(16.1)$ & $3(9.7)$ & $1(3.2)$ & $0(0.0)$ & \\
\hline Surgical academicians & $0(0.0)$ & $4(12.9)$ & $1(3.2)$ & $0(0.0)$ & $0(0.0)$ & \\
\hline $\begin{array}{l}\text { Distance education lessons are as useful } \\
\text { as face-to-face lessons }\end{array}$ & & & & & & 0.007 \\
\hline Preclinical academicians & $0(0.0)$ & $1(3.2)$ & $0(0.0)$ & $0(0.0)$ & $1(3.2)$ & \\
\hline Medical academicians & $10(32.3)$ & $4(12.9)$ & $6(19.4)$ & $4(12.9)$ & $0(0.0)$ & \\
\hline Surgical academicians & $0(0.0)$ & $1(3.2)$ & $3(9.7)$ & $1(3.2)$ & $0(0.0)$ & \\
\hline $\begin{array}{l}\text { I would like distance education to be } \\
\text { available also outside of working hours }\end{array}$ & & & & & & 0.180 \\
\hline Preclinical academicians & $0(0.0)$ & $1(3.2)$ & $0(0.0)$ & $1(3.2)$ & $0(0.0)$ & \\
\hline Medical academicians & $6(19.4)$ & $5(16.1)$ & $6(19.4)$ & $7(22.6)$ & $0(0.0)$ & \\
\hline Surgical academicians & $4(12.9)$ & $1(3.2)$ & $0(0.0)$ & $0(0.0)$ & $0(0.0)$ & \\
\hline $\begin{array}{l}\text { The theoretical courses is suitable for } \\
\text { distance education }\end{array}$ & & & & & & 0.382 \\
\hline Preclinical academicians & $0(0.0)$ & $0(0.0)$ & $0(0.0)$ & $1(3.2)$ & $1(3.2)$ & \\
\hline Medical academicians & $0(0.0)$ & $5(16.1)$ & $8(25.8)$ & $9(29.0)$ & $2(6.5)$ & \\
\hline Surgical academicians & $0(0.0)$ & $1(3.2)$ & $3(9.7)$ & $1(3.2)$ & $0(0.0)$ & \\
\hline $\begin{array}{l}\text { Practical lessons are suitable for distance } \\
\text { education }\end{array}$ & & & & & & 0.026 \\
\hline Preclinical academicians & $1(3.2)$ & $0(0.0)$ & $0(0.0)$ & $0(0.0)$ & $1(3.2)$ & \\
\hline Medical academicians & $15(48.4)$ & $6(19.4)$ & $2(6.5)$ & $1(3.2)$ & $0(0.0)$ & \\
\hline Surgical academicians & $3(9.7)$ & $1(3.2)$ & $0(0.0)$ & $1(3.2)$ & $0(0.0)$ & \\
\hline $\begin{array}{l}\text { Apart from the pandemic, theoretical } \\
\text { lessons can be given in the form of } \\
\text { distance education }\end{array}$ & & & & & & 0.065 \\
\hline Preclinical academicians & $0(0.0)$ & $0(0.0)$ & $0(0.0)$ & $1(3.2)$ & $1(3.2)$ & \\
\hline Medical academicians & $11(35.5)$ & $3(9.7)$ & $4(12.9)$ & $5(16.1)$ & $1(3.2)$ & \\
\hline Surgical academicians & $0(0.0)$ & $1(3.2)$ & $3(9.7)$ & $1(3.2)$ & $0(0.0)$ & \\
\hline
\end{tabular}

*Statistically significant values are marked as bold. 
Table 6: Thoughts of clinical vs. pre-clinical students and academicians on distance education sufficiency.

\begin{tabular}{|c|c|c|c|c|c|c|c|}
\hline & \multicolumn{3}{|c|}{ Medical Students } & \multicolumn{4}{|c|}{ Academicians } \\
\hline & $\begin{array}{c}\text { Preclinical } \\
\text { Students } \\
\text { n= 165 } \\
(100 \%)\end{array}$ & $\begin{array}{c}\text { Clinical } \\
\text { Students } \\
\mathbf{n}=\mathbf{1 2 5} \\
(\mathbf{1 0 0} \%)\end{array}$ & P-value* & $\begin{array}{c}\text { Preclini } \\
\text { cal } n=2 \\
(100 \%)\end{array}$ & $\begin{array}{c}\text { Medical } \\
n=24 \\
(100 \%)\end{array}$ & $\begin{array}{c}\text { Surgical } \\
n=5 \\
(100 \%)\end{array}$ & P-value \\
\hline $\begin{array}{l}\text { The competence of distance } \\
\text { education for basic sciences }\end{array}$ & & & $<0.001$ & & & & 0.199 \\
\hline Alone enough & $22(13.3)$ & $27(21.6)$ & & $1(50.0)$ & $3(12.5)$ & $1(20.0)$ & \\
\hline $\begin{array}{l}\text { Inadequate alone, but most of them } \\
\text { should be distance education }\end{array}$ & $63(38.2)$ & $71(56.8)$ & & $0(0.0)$ & $12(50.0)$ & $0(0.0)$ & \\
\hline $\begin{array}{l}\text { Inadequate alone, and most of them } \\
\text { should be in-class education }\end{array}$ & $58(35.2)$ & $26(20.8)$ & & $0(0.0)$ & $7(29.2)$ & $4(80.0)$ & \\
\hline $\begin{array}{l}\text { Completely insufficient alone, all } \\
\text { them should be in-class education }\end{array}$ & $22(13.3)$ & $1(0.8)$ & & $1(50.0)$ & $2(8.3)$ & $0(0.0)$ & \\
\hline $\begin{array}{l}\text { The competence of distance } \\
\text { education for clinical sciences }\end{array}$ & & & 0.011 & & & & 0.078 \\
\hline Alone enough & $2(1.2)$ & $4(3.2)$ & & $1(50.0)$ & $0(0.0)$ & $1(20.0)$ & \\
\hline $\begin{array}{l}\text { Inadequate alone, but most of them } \\
\text { should be distance education }\end{array}$ & $13(7.9)$ & $22(17.6)$ & & $0(0.0)$ & $3(12.5)$ & $0(0.0)$ & \\
\hline $\begin{array}{l}\text { Inadequate alone, and most of them } \\
\text { should be in-class education }\end{array}$ & $66(40.0)$ & $55(44.0)$ & & $0(0.0)$ & $14(58.3)$ & $3(60.0)$ & \\
\hline $\begin{array}{l}\text { Completely insufficient alone, all of } \\
\text { them should be in-class education }\end{array}$ & $84(50.9)$ & $44(35.2)$ & & $1(50.0)$ & $7(29.2)$ & $1(20.0)$ & \\
\hline
\end{tabular}

*Statistically significant values are marked as bold. 
Table 7: The students' user experience of distance learning applications.

\begin{tabular}{|c|c|c|c|c|c|c|}
\hline & $\begin{array}{l}\text { Do not } \\
\text { agree at all } \\
\mathbf{n}(\%)\end{array}$ & $\begin{array}{c}\text { Do not } \\
\text { agree } \\
\text { n (\%) }\end{array}$ & $\begin{array}{l}\text { Indecisive } \\
\text { n (\%) }\end{array}$ & $\begin{array}{l}\text { Agree } \\
\text { n (\%) }\end{array}$ & $\begin{array}{c}\text { Completely } \\
\text { agree } \\
\text { n (\%) }\end{array}$ & $\begin{array}{c}\text { P- } \\
\text { value } \\
*\end{array}$ \\
\hline $\begin{array}{l}\text { Accessing the course is easy in the } \\
\text { distance education system }\end{array}$ & & & & & & 0.046 \\
\hline Preclinical Students & $12(4.1)$ & $27(9.3)$ & $32(11.0)$ & $71(24.5)$ & $23(7.9)$ & \\
\hline Clinical Students & $2(0.7)$ & $12(4.1)$ & $23(7.9)$ & $70(24.1)$ & $18(6.2)$ & \\
\hline $\begin{array}{l}\text { I would like distance education to be } \\
\text { available also outside of working hours }\end{array}$ & & & & & & 0.540 \\
\hline Preclinical Students & $42(14.5)$ & $43(14.8)$ & $33(11.4)$ & $32(11.0)$ & $15(5.2)$ & \\
\hline Clinical Students & $32(11.0)$ & $29(10.0)$ & $32(11.0)$ & $26(9.0)$ & $6(2.1)$ & \\
\hline $\begin{array}{l}\text { Accessing and watching the recordings of } \\
\text { the live lectures I did not attend are easy }\end{array}$ & & & & & & 0.006 \\
\hline $\begin{array}{l}\text { Preclinical Students } \\
\text { Clinical Students }\end{array}$ & $\begin{array}{c}11(3.8) \\
3(1.0)\end{array}$ & $\begin{array}{l}16(5.5) \\
16(5.5)\end{array}$ & $\begin{array}{l}50(17.2) \\
19(6.6)\end{array}$ & $\begin{array}{l}58(20.0) \\
64(22.1)\end{array}$ & $\begin{array}{c}30(10.3) \\
23(7.9)\end{array}$ & \\
\hline $\begin{array}{l}\text { The educational materials used in the } \\
\text { lessons meet my needs }\end{array}$ & & & & & & 0.062 \\
\hline Preclinical Students & $22(7.6)$ & $40(13.8)$ & $51(17.6)$ & $39(13.4)$ & $13(4.5)$ & \\
\hline Clinical Students & $5(1.7)$ & $27(9.3)$ & $51(17.6)$ & $31(10.7)$ & $11(3.8)$ & \\
\hline I can quickly access the course contents & & & & & & 0.027 \\
\hline Preclinical Students & $8(2.8)$ & $23(7.9)$ & $35(12.1)$ & $79(27.2)$ & $20(6.9)$ & \\
\hline Clinical Students & $2(0.7)$ & $12(4.1)$ & $14(4.8)$ & $75(25.9)$ & $22(7.6)$ & \\
\hline $\begin{array}{l}\text { I know how to solve the problems I } \\
\text { encounter in the distance education } \\
\text { system }\end{array}$ & & & & & & 0.135 \\
\hline $\begin{array}{l}\text { Preclinical Students } \\
\text { Clinical Students }\end{array}$ & $\begin{array}{c}19(6.6) \\
7(2.4)\end{array}$ & $\begin{array}{l}39(13.4) \\
21(7.2)\end{array}$ & $\begin{array}{l}54(18.6) \\
52(17.9)\end{array}$ & $\begin{array}{l}41(14.1) \\
38(13.1)\end{array}$ & $\begin{array}{c}12(4.1) \\
7(2.4)\end{array}$ & \\
\hline $\begin{array}{l}\text { I know how to use virtual classroom } \\
\text { application }\end{array}$ & & & & & & 0.151 \\
\hline Preclinical Students & $24(8.3)$ & $35(12.1)$ & $37(12.8)$ & $55(19.0)$ & $14(4.8)$ & \\
\hline Clinical Students & $11(3.8)$ & $23(7.9)$ & $22(7.6)$ & $50(17.2)$ & $19(6.6)$ & \\
\hline $\begin{array}{l}\text { I occasionally encounter technical } \\
\text { problems in virtual classroom application }\end{array}$ & & & & & & 0.017 \\
\hline Preclinical Students & $10(3.4)$ & $17(5.9)$ & $69(23.8)$ & $53(18.3)$ & $16(5.5)$ & \\
\hline Clinical Students & $13(4.5)$ & $19(6.6)$ & $37(12.8)$ & $52(17.9)$ & $4(1.4)$ & \\
\hline $\begin{array}{l}\text { The distance education system seems very } \\
\text { complicated to me }\end{array}$ & & & & & & 0.021 \\
\hline Preclinical Students & $31(10.7)$ & $60(20.7)$ & $29(10.0)$ & $27(9.3)$ & $18(6.2)$ & \\
\hline Clinical Students & $36(12.4)$ & $55(19.0)$ & $17(5.9)$ & $12(4.1)$ & $5(1.7)$ & \\
\hline $\begin{array}{l}\text { Finding the information I want in the } \\
\text { distance education system is easy }\end{array}$ & & & & & & 0.532 \\
\hline Preclinical Students & $17(5.9)$ & $33(11.4)$ & $53(18.3)$ & $49(16.9)$ & $13(4.5)$ & \\
\hline Clinical Students & $6(2.1)$ & $26(9.0)$ & $40(13.8)$ & $42(14.5)$ & $11(3.8)$ & \\
\hline $\begin{array}{l}\text { The information (announcement etc.) is } \\
\text { sufficient }\end{array}$ & & & & & & 0.298 \\
\hline Preclinical Students & $31(10.7)$ & $38(13.1)$ & $28(9.7)$ & $50(17.2)$ & $18(6.2)$ & \\
\hline Clinical Students & $22(7.6)$ & $35(12.1)$ & $27(9.3)$ & $35(12.1)$ & $6(2.1)$ & \\
\hline
\end{tabular}

*Statistically significant values are marked as bold. 
Table 8: The academicians' user experience of distance learning applications.

\begin{tabular}{|c|c|c|c|c|c|c|}
\hline & $\begin{array}{c}\text { Do not } \\
\text { agree at all } \\
\text { n (\%) }\end{array}$ & $\begin{array}{l}\text { Do not } \\
\text { agree } \\
\text { n (\%) }\end{array}$ & $\begin{array}{l}\text { Indecisive } \\
\text { n (\%) }\end{array}$ & $\begin{array}{l}\text { Agree } \\
\text { n (\%) }\end{array}$ & $\begin{array}{l}\text { Completely } \\
\text { agree } \\
\text { n (\%) }\end{array}$ & $\begin{array}{l}\text { P-value } \\
*\end{array}$ \\
\hline $\begin{array}{l}\text { I know how to solve the problems I } \\
\text { encounter in the distance education } \\
\text { system }\end{array}$ & & & & & & 0.000206 \\
\hline Preclinical academicians & $0(0.0)$ & $0(0.0)$ & $1(3.2)$ & $0(0.0)$ & $1(3.2)$ & \\
\hline Medical academicians & $0(0.0)$ & $7(22.6)$ & $11(35.5)$ & $6(19.4)$ & $0(0.0)$ & \\
\hline Surgical academicians & $0(0.0)$ & $0(0.0)$ & $0(0.0)$ & $5(16.1)$ & $0(0.0)$ & \\
\hline $\begin{array}{l}\text { I know how to use virtual classroom } \\
\text { application without support }\end{array}$ & & & & & & 0.181 \\
\hline Preclinical academicians & $0(0.0)$ & $0(0.0)$ & $0(0.0)$ & $1(3.2)$ & $1(3.2)$ & \\
\hline Medical academicians & $0(0.0)$ & $4(12.9)$ & $10(32.3)$ & $9(29.0)$ & $1(3.2)$ & \\
\hline Surgical academicians & $0(0.0)$ & $0(0.0)$ & $3(9.7)$ & $2(6.5)$ & $0(0.0)$ & \\
\hline $\begin{array}{l}\text { I occasionally encounter technical } \\
\text { problems in virtual classroom application }\end{array}$ & & & & & & 0.681 \\
\hline Preclinical academicians & $0(0.0)$ & $0(0.0)$ & $1(3.2)$ & $1(3.2)$ & $0(0.0)$ & \\
\hline Medical academicians & $0(0.0)$ & $2(6.5)$ & $8(25.8)$ & $12(38.7)$ & $2(6.5)$ & \\
\hline Surgical academicians & $0(0.0)$ & $1(3.2)$ & $0(0.0)$ & $4(12.9)$ & $0(0.0)$ & \\
\hline $\begin{array}{l}\text { The distance education system seems very } \\
\text { complicated to me }\end{array}$ & & & & & & 0.186 \\
\hline Preclinical academicians & $1(3.2)$ & $1(3.2)$ & $0(0.0)$ & $0(0.0)$ & $0(0.0)$ & \\
\hline Medical academicians & $4(12.9)$ & $9(29.0)$ & $7(22.6)$ & $4(12.9)$ & $0(0.0)$ & \\
\hline Surgical academicians & $0(0.0)$ & $5(16.1)$ & $0(0.0)$ & $0(0.0)$ & $0(0.0)$ & \\
\hline $\begin{array}{l}\text { The distance education lessons are } \\
\text { interactive enough }\end{array}$ & & & & & & 0.007 \\
\hline Preclinical academicians & $1(3.2)$ & $0(0.0)$ & $0(0.0)$ & $0(0.0)$ & $1(3.2)$ & \\
\hline Medical academicians & $6(19.4)$ & $10(32.3)$ & $6(19.4)$ & $2(6.5)$ & $0(0.0)$ & \\
\hline Surgical academicians & $0(0.0)$ & $4(12.9)$ & $0(0.0)$ & $1(3.2)$ & $0(0.0)$ & \\
\hline
\end{tabular}

¥Statistically significant values are marked as bold.

Table 9: Clinical vs. pre-clinical students and academicians on online examinations.

\begin{tabular}{|c|c|c|c|c|c|c|}
\hline & $\begin{array}{c}\text { Do not } \\
\text { agree at all } \\
\text { n (\%) } \\
\end{array}$ & $\begin{array}{c}\text { Do not } \\
\text { agree } \\
\text { n (\%) } \\
\end{array}$ & $\begin{array}{c}\text { Indecisive } \\
\text { n (\%) }\end{array}$ & $\begin{array}{l}\text { Agree } \\
\text { n (\%) }\end{array}$ & $\begin{array}{c}\text { Completely } \\
\text { agree } \\
\text { n (\%) } \\
\end{array}$ & $\begin{array}{c}\text { P-value } \\
*\end{array}$ \\
\hline \multicolumn{7}{|l|}{ Medical Students } \\
\hline Online exams measure learning & & & & & & 0.983 \\
\hline Preclinical Students & $60(20.7)$ & $25(8.6)$ & $30(10.3)$ & $34(11.7)$ & $16(5.5)$ & \\
\hline Clinical Students & $45(15.5)$ & $22(7.6)$ & $23(7.9)$ & $24(8.3)$ & $11(3.8)$ & \\
\hline Online exams are reliable & & & & & & 0.108 \\
\hline Preclinical Students & $60(20.7)$ & $42(14.5)$ & $19(6.6)$ & $27(9.3)$ & $17(5.9)$ & \\
\hline Clinical Students & $38(13.1)$ & $25(8.6)$ & $29(10.0)$ & $21(7.2)$ & $12(4.1)$ & \\
\hline \multicolumn{7}{|l|}{ Academicians } \\
\hline Online exams measure learning & & & & & & 0.000239 \\
\hline Preclinical academicians & $1(3.2)$ & $0(0.0)$ & $0(0.0)$ & $0(0.0)$ & $1(3.2)$ & \\
\hline Medical academicians & $7(22.6)$ & $8(25.8)$ & $8(25.8)$ & $1(3.2)$ & $0(0.0)$ & \\
\hline Surgical academicians & $0(0.0)$ & $2(6.5)$ & $0(0.0)$ & $3(9.7)$ & $0(0.0)$ & \\
\hline Online exams are reliable & & & & & & 0.003 \\
\hline Preclinical academicians & $1(3.2)$ & $0(0.0)$ & $0(0.0)$ & $0(0.0)$ & $1(3.2)$ & \\
\hline Medical academicians & $8(25.8)$ & $11(35.5)$ & $4(12.9)$ & $1(3.2)$ & $0(0.0)$ & \\
\hline Surgical academicians & $0(0.0)$ & $1(3.2)$ & $3(9.7)$ & $1(3.2)$ & $0(0.0)$ & \\
\hline $\begin{array}{l}\text { I think the students cheat in the } \\
\text { online exams }\end{array}$ & & & & & & 0.027 \\
\hline Preclinical academicians & $0(0.0)$ & $1(3.2)$ & $0(0.0)$ & $0(0.0)$ & $1(3.2)$ & \\
\hline Medical academicians & $0(0.0)$ & $2(6.5)$ & $3(9.7)$ & $16(51.6)$ & $3(9.7)$ & \\
\hline Surgical academicians & $0(0.0)$ & $1(3.2)$ & $3(9.7)$ & $0(0.0)$ & $1(3.2)$ & \\
\hline
\end{tabular}

${ }^{*}$ Statistically significant values are marked as bold. 


\section{DISCUSSION}

Since the COVID-19 outbreak, this pandemic has been a turning point for medical schools and medical students at many points (4). Universities have been closed all over the world. Many universities have turned face-to-face education methods into online education or a combination of online and traditional education (7). Online education suddenly became an academic principle. Experts predicted that it would take 5-10 years to recover from this pandemic (8). Therefore, it is important to identify the thoughts and opinions of medical students and academicians about the online education and examination system that is rapidly passed through the COVID-19 pandemic process. In this study, 290 medical students and 31 academicians from different medical schools in Turkey filled out an online survey, and results were evaluated descriptively.

Most of the students stated that they followed the online education lessons with computers. Computers played a major role in social distancing and rapid innovation, especially during the pandemic period (9). Many respondents (49\% agree, $28.3 \%$ strongly agree) attended their online lessons. About 40\% (26.9\% do not agree, $13.4 \%$ do not agree at all) said online courses are useless, while $29.7 \%$ of students are indecisive about whether online courses are useful. Only about $9.3 \%$ of them (4.5\% agree, $4.8 \%$ completely agree) found it sufficient, but $64.1 \%$ of the students stated that they did not find online education alone sufficient in the field of medicine. The fact that practical lessons occupy a large place and are very important in medical education may have been effective in this decision. For example, laboratory lessons are difficult or impossible to adapt to the virtual environment. However, according to Rajab et al. (8), 67\% of Saudi Arabian medical students stated that the pandemic had a positive effect on online learning. Most of the students (40.7\% do not agree at all, 25.9\% do not agree) stated that online education classes are not as useful as face-to-face lessons. However, in the academic community, hybrid education has started to gain more acceptance than face-to-face education because it combines the best features of the two types of education (8).

Most of students can easily access the courses and course materials within the scope of distance education: $48.5 \%$ of the students stated that they can easily access the course they want in the distance education system, while $52.9 \%$ stated that they can access the course contents quickly. However, the majority of students with a rate of $35.1 \%$ declared that they were indecisive that the educational materials used in the courses were enough for their needs. This can be explained by the fact that each school follows different individual paths for distance education and the course materials may vary between schools and classes. According to the study done by Srinivasan (10), different platforms such as Zoom, PollEV can be used for e-learning. Besides in the study of Chick et al. (5), it was found that social media platforms like Facebook groups and video archives of the medical associations such as the American College of Surgeons can be used as educational materials for distance education as well.

Students could easily access and watch the recordings of the live classes that they did not attend on time, $60.1 \%$ of the students (18.2\% strongly agree, $41.9 \%$ agree) stated. Despite this, almost half of the students (25.8\% strongly disagree and $24.7 \%$ disagree) have a negative opinion on that they would like distance education to be available also outside of working hours. This quandary, that the students watch the recordings of lessons at their own time while not wanting distance education to be done outside of working hours, shows that the only function of face-to-face education is not only to attend live classes but at the same time, it provides a suitable environment for learning. Students who remained in isolation at home had difficulties in setting effective boundaries between home and school during COVID-19 (11).

Furthermore, $85.6 \%$ of students think that the practical lessons are not suitable for distance education, while $63.6 \%$ of which were strong on their opinion. Besides, $44.3 \%$ of the students believe that distance education is completely inadequate by itself, all the training should be face-to-face. Considering that most of the clinical sciences consist of practical courses, students especially attach importance to experience practical skills in clinics under the supervision of clinicians. Parallel to that, $64.3 \%$ of the students (43.3\% strongly disagree, and $21 \%$ disagree) declared that "they find it incorrect to include interns in distance education" concerning the 6th-grade internship education, almost all of which are based on practical applications. In addition, $52.2 \%$ of the students expressed that the theoretical courses are suitable for distance education while $18.9 \%$ of which strongly agreed. However, $46 \%$ of the students believe that distance education is insufficient alone for the basic medical sciences, but most of the education in this field should be distant. Even though, the students answered "strongly disagree" with 33.3\% and "disagree" with $19.9 \%$ to the question "I think that theoretical courses can be conducted in the form of 
distance education even outside the pandemic process". This can be interpreted by considering that theoretical courses are taught with more conventional methods than practical courses and as a matter of fact that basic medical sciences are mostly composed of theoretical courses, basic medical sciences are more convenient to distance education. If the materials used in distance education can be made more diverse especially for practical courses and hybrid education models are used more widely, distance education may have positive results for students with different learning speeds and styles (12).

Applied courses are not required by students to be conducted online. Considering that the most important part of medical education is practicing on the patient, it is not surprising that this opinion is the majority. The research of Ruiz et al. (13) also supports this idea.

The biggest problem faced by the participants during their participation in online classes is seen as the inability to provide a suitable course environment. This affects the success of the students and also decreases their motivation to be interactive (14). Approximately $10 \%$ of the participants stated that they do not know how to use virtual applications and cannot solve if a problem occurs in the system. Compared to the literature, this outcome is not out of normal range (15).

The vast majority of students do not have difficulties with virtual classroom applications, Buckley et al. (16) stated that this is not a surprising outcome due to high access to the technology. Easy access to the information sought in distance education systems was avetaged by the students. It has been stated in many researches that the infrastructure and site designs are very effective at this point (14).

Although the majority of the students think that they are sufficient in informing, the differences in access to technology also affect the informational effectiveness (16). Although the majority of the students think that they are sufficient in informing, the differences in access to technology also affect the informational effectiveness (15).

Almost $1 / 3$ of the participants thought that the exam was insufficient in measuring their knowledge level, but there was no difference in the scale of the exams taken in the classroom or online in the studies because nowadays most medical schools prefer test system in both cases (17).

When asked about the reliability of the exam, 33\% of the participants stated that they did not find the exam reliable, but Buckley et al. (16) did not find a significant difference in reliability between online and paper exams in their study. However, the differences in exam systems and departments require a lot of new and large-scale studies to be done in this area.

A 2014 study, long before the pandemic, looked into the use of technology in education (18). One of the biggest technostress risk factors they found was age. They claimed the elder academicians experienced more stress using the online applications required for distance learning. Meanwhile the younger academicians supported the application of distance learning. The mean age of the participants in our study was 41.68 years \pm 7.51 , which is classified as young in the 2014 study (18). This could explain the relative ease in using the distance education applications in the academician group. However, contrary to that study, we found a majority of the academicians were not leaning toward distance education despite the ease of use. In addition, there was no difference between academic experience groups in terms of preferring distance education applications; disproving the idea of more experienced academicians being more traditional.

The majority of the academicians $[n=23(74.2 \%)]$ did not suffer from any chronical illnesses. In any case there was no significant difference between those who suffered from chronical illnesses and those who did not despite a greatly increased risk in terms of COVID-19 infection and prognosis $(19,20)$.

There is a split decision when it comes to distance lessons being beneficial. Eleven agreed, 12 disagreed, and 8 were indecisive. Academicians in the surgical field thought it was beneficial compared to other branches. The study conducted in Alfaisal University revealed that the $78.9 \%$ of the academicians thought the distance education was beneficial for the students (8). Interestingly this ratio was even higher than the students. Only $66.9 \%$ of the students thought distance education was beneficial (8). In our study $30 \%$ of the students thought online education was beneficial. The reasons behind the students preferring the more traditional face-to-face lessons should be evaluated. However, when the academicians were asked whether they find distance education alone sufficient in the field of medicine the decision was more in unity. Twenty-seven disagreed among which 18 strongly disagreed. The results show that the idea of medical education being a "master-apprentice relationship" is well accepted among the academicians.

The academicians did not want the interns to be a part of the distance education program. Some countries even proposed and practiced graduating the interns early to distribute the workload (21-23). We did not question whether the academicians would prefer the interns to be a part of the workforce in the medical field with early graduations. However, they would pre- 
fer the interns to be a part of the face-to-face education where they work as practitioners under the supervision of the academicians. Academicians may have answered this question thinking that interns should work in hospitals to acquire the necessary practical skills in their professional life, since they are in the last year of their education. When compared with face to face lessons, $51.6 \%$ of the academicians said the distance education lessons were not as good. This is similar to the results of Alfaisal University study where only $12 \%$ of the participants thought online lessons alone could replace faceto-face lessons (8). The clinical branches disagree more compared to the preclinical branches. Considering the theoretical disposition of the preclinical branches, this result is expected.

The majority of the academicians disagreed when it came to distance education outside of working hours and both clinical and preclinical branches agreed on the topic. The pandemic caused healthcare workers to take extra shifts and work longer hours (24). Even the preclinical branches were called for duty in Turkey to help alleviate the heavy workload. Considering the already long working hours, it is understandable that the academicians do not want to extend distance education outside of work.

Academicians were more inclined towards distance education when the theoretical lessons were considered. Only 6 (19.4\%) disagreed with the statement; "The theoretical courses are suitable for distance education" and none of the academicians completely disagreed. However, when it came to practical lessons 26 disagreed with 19 of them completely disagreeing. Yardley et al. (25) claims the transition from a freshman medical student to a qualified doctor was based more on experiential learning.

The majority of academicians thought distance education in the field of medicine was not enough by itself. While we compare the clinical and theoretical education, academicians predominately support face-to-face learning methodology for clinical education. This may be associated with reduced learning of clinical skills that model future physicians. This also explains why academicians find it inconvenient to include interns in this education model. However, some studies claim that effective distance clinical education is possible in favorable conditions. Marshall et al. (26) reported that including clinical students in tele-interactions with patients, using artificial intelligence, and receiving consultancy from trained clinicians can develop a positive distance learning environment. Usage of webinars, online atlases, simulations, and 3D models can be complementary to distance clinical and theoretical educa- tion to create a learner-centric manner (27).

On continuing theoretical lessons online after the pandemic, academicians mostly opposed the idea like the students. However, in our study, one of five academicians were hesitant who may adapt to change later by blended learning system as a complement to increase efficiency and effectiveness in their in-class lessons (13). For instance, a study conducted at the University of Dubai recommends continuing to offer a blended learning system after the pandemic (28). In another study, both students and faculty members agreed that online education is an effective way of learning, and teaching that includes resource, and time efficiency (29).

Most of the academicians are indecisive about whether they are capable of solving the problems they encountered or not. Following that option, a similar ratio was seen in the group that knew how to solve those problems. The results were similar between indecisive and agreed groups on knowing how to use the virtual classroom application without support. Despite this implication, more than $60 \%$ of the academicians encounter technical problems in virtual classroom applications. The problems academicians faced were slightly the same as the students, but in ratio, they had lesser difficulties. Assuming that effective internet connectivity is available to every student was wrong (30). The same case can be adapted to the academicians because relying on the idea that each of them has their uninterrupted connection is not accurate (31). They can experience systemic issues or computer related problems during lectures even though they use the university's facility.

More than half of the academicians predicated that virtual lessons were not interactive enough. The impact of asynchronous content delivery may result in a lack of communication (13). Instead, using real-time learning and instant transmission of questions can help to overcome this (13). Nevertheless, the virtual lessons and online examinations are part of the current learning system whether they are qualified or not to prevent more positive cases. However, when it comes to their measuring standards and reliability, online examinations may create doubt in academicians. Only around $16 \%$ of the academicians think online exams are measuring students' knowledge accurately. Contrary, Longhurst et al. (32) claim that preventing cheating, and improving examination conditions are possible to design traditional-like online examinations to measure knowledge accurately. On the question of reliability, not reliable (38.7\%) followed by completely not reliable (29\%) were the most selected options. Besides, $67.7 \%$ 
of them think that students were cheating in the online exams. Schmidt et al. (33) had the same concern in their case study so they created unique exams for each student, but emphasized that even if the exam was not random, universities have their honor systems on not cheating.

Twenty-four medical, five surgical specialty academicians participated representing the clinical years. However, there were only two preclinical academicians who participated in the survey which is thought to be the greatest limitation of this study.

In conclusion, it is predicted that the pandemic process will continue in the next academic years. Considering the current situation, distance education seems to be the best option to ensure that the learning process can continue while protecting the health of students and academicians. Although distance education is not sufficient by itself in medical education during and after the COVID-19 pandemic, it is a method that should be used in almost every field of medical education, especially in the preclinical phase. Both the distance education, and online examination process require improvements and they are needed to be supported with face to face lectures and practices.

Ethics Committee Approval: This study was approved by the Scientific Research Ethical Committee of Trakya University School of Medicine (Protocol Code: TÜTF-BAEK 2020/343).

Informed Consent: Online informed consent was obtained from the participants of this study.

Conflict of Interest: The authors declared no conflict of interest. Author contributions: Concept: BK, HSÇ, IIÖ, SS, AG, SGS, SA. Design: IİÖ, BK, HSÇ, SS, AG, SGS, SA. Supervision: SA, SGS, BK, HSÇ, IIÖ, SS, AG. Resources: HSÇ, IİÖ, BK, SS, AG, SGS, SA. Materials: IIIÖ, BK, HSÇ, SS, AG, SGS, SA. Data collection and/or processing: BK, HSÇ, IIIÖ, SS, AG, SGS, SA. Analysis and/or Interpretation: IİÖ, HSÇ, BK, SS, AG, SGS, SA. Literature Search: IIIÖ, HSÇ, BK, SS, AG, SGS, SA. Writing Manuscript: IİÖ, HSÇ, BK, SS, AG, SGS, SA. Critical Review: SA, SGS, BK, HSÇ, IİÖ, SS, AG. Financial disclosure: The authors declared that this study received no financial support.

Editor in chief's Note: Five of the authors of this article, Hilal Sena Çifcibaşı, Berkay Kef, Irmak İrem Özyiğit, Sezin Sayın, and Aslı Göztepe are members of the editorial board of Turkish Medical Student Journal. However, they did not take place in any stage of the editorial decision of the manuscript. The editors who evaluated this manuscript are from other institutions.

\section{REFERENCES}

1. Cascella M, Rajnik M, Cuomo A et al. Features, evaluation, and treatment of coronavirus (COVID-19). In: StatPearls (serial online) 2020 Oct (cited 2020 October 4). Treasure Island (FL): StatPearls Publishing; 2020. Available from: URL:https://www.ncbi.nlm.nih. gov/books/NBK554776/.
2. Thu TPB, Ngoc PNH, Hai NM et al. Effect of social distancing measures on the spread of COVID-19 in 10 highly infected countries. Sci Total Environ 2020;742:140430.

3. Demirbilek Y, Pehlivantürk G, Özgüler ZÖ et al. COVID-19 outbreak control, example of ministry of health of Turkey. Turk J Med Sci 2020;50:489-94.

4. Ferrel MN, Ryan JJ. The impact of COVID-19 on medical education. Cureus 2020;12:7492.

5. Chick RC, Clifton GT, Peace KM et al. Using technology to maintain the education of residents during the COVID-19 pandemic. J Surg Educ 2020;77:729-32.

6. Singh K, Srivastav S, Bhardwaj A et al. Medical education during the COVID-19 pandemic: a single institution experience. Indian Pediatr 2020;57:678-9.

7. Calhaun KH, Yale LA, Whipple M et al. The impact of COVID-19 on medical student surgical education: Implementing extreme pandemic response measures in a widely distributed surgical clerkship experience. Am J Surg 2020;220(1):44-7.

8. Rajab MH, Gazal AM, Alkattan K. Challenges to online medical education during the COVID-19 pandemic. Cureus 2020;12(7):e8966.

9. Radanliev P, Roure DD, Walton R et al. COVID-19 what have we learned? The rise of social machines and connected devices in pandemic management following the concepts of predictive, preventive and personalized medicine. EPMA J 2020;11(3):311-32.

10. Srinivasan DK. Medical students' perceptions and an anatomy teacher's personal experience using an e-learning platform for tutorials during the COVID-19 crisis. Anat Sci Educ 2020;13(3):318-9. 11. Rose S. Medical student education in the time of COVID-19. JAMA 2020;323(21):2131-2.

12. Ozer MA, Govsa F, Bati AH. Web-based teaching video packages on anatomical education. Surg Radiol Anat 2017;39(11):1253-61. 13. Ruiz JG, Mintzer MJ, Leipzig RM. The impact of e-learning in medical education. Academic Medicine 2006;81(3):207-12.

14. Coulehan J, Williams P. Vanquishing virtue: The impact of medical education. Academic Medicine 2001;76(6):598-605.

15. Stowell JR, Bennett D. Effects of online testing on student exam performance and test anxiety. Journal of Educational Computing Research 2010;42(2):161-71.

16. Buckley J, Canty D, Seery N. The validity and reliability of online testing for the assessment of spatial ability. ASEE Conference Oct 2016.

17. Harmon O, Lambrinos J. Are online exams an invitation to cheat? The Journal of Economic Education 2008;39(2):116-25.

18. Jena RK, Mahanti K. An empirical study of technostress among Indian academicians. International Journal of Education and Learning 2014;3(2):1-10.

19. Kang C, Yang S, Yuan J et al. Patients with chronic illness urgently need integrated physical and psychological care during the COVID-19 outbreak. Asian Journal of Psychiatry 2020;51(6): 102081.

20. Sanyaolu A, Okorie C, Marinkovic A et al. Comorbidity and its impact on patients with COVID-19. SN Comprehensive Clinical Medicine 2020;25(6);1-8.

21. Sharif SP. UK medical students graduating early to work during the COVID-19 pandemic. Psychological Medicine 2020;5(7):1-4.

22. Harvey A. Covid-19: Medical schools given powers to graduate final year students early to help NHS. BMJ 2020;368:1227.

23. Flotte TR, Larkin AC, Fischer MA et al. Accelerated graduation and the deployment of new physicians during the COVID-19 pandemic. Acad Med 2020;95(10):1492-4.

24. Gupta B, Bajwa SJS, Malhotra N et al. Tough times and miles to go before we sleep-corona warriors. Indian Journal of Anaesthesia 2020;64(14):120-4. 
25. Yardley S, Teunissen PW, Dornan T. Experiential learning: Transforming theory into practice. Medical Teacher 2012;34(2):161-4. 26. Marshall AL, Wolanskyj-Spinner A. COVID-19: Challenges and opportunities for educators and generation Z learners. Mayo Clin Proc 2020;95(6):1135-7.

27. Dedeilia A, Sotiropoulos MG, Hanrahan JG et al. Medical and surgical education challenges and innovations in the COVID-19 era: A systematic review. In Vivo 2020;34(3):1603-11.

28. Almuraqab N. Shall universities at the UAE continue distance learning after the COVID-19 pandemic? Revealing students' perspective. IJARET 2020;11(5):226-33.

29. Mukhtar K, Javed K, Arooj M et al. Advantages, limitations and recommendations for online learning during COVID-19 pandemic era. Park J Med Sci 2020;36:27-31.

30. COVID-19 and higher education: Today and tomorrow. Impact analysis, policy responses and recommendations by IESALC. 2020. 31. Yusoff MSB, Hadie SNH, Mohamad I et al. Sustainable medical teaching and learning during the COVID-19 pandemic: Surviving the new normal. Malays J Med Sci 2020;27(3):137-42.

32. Longhurst GJ, Stone DM, Dulohery K et al. Strength, weakness, opportunity, threat (SWOT) analysis of the adaptations to anatomical education in the United Kingdom and Republic of Ireland in response to the Covid-19 pandemic. Anat Sci Educ 2020;13:298-308. 33. Schmidt SMP, Ralph DL, Buskirk B. Utilizing online exams: A case study. Journal of College Teaching \& Learning 2009;6(8):1-8. 